OPEN ACCESS

Edited by:

Cheryl Ann Winkler,

Frederick National Laboratory for Cancer Research (NIH),

United States

Reviewed by:

José Ascención Martínez-Álvarez, Universidad de Guanajuato, Mexico Masaaki Miyazawa

Kindai University, Japan

*Correspondence.

Valli $\mathrm{De} R$

vdere@cro.it

†These authors have contributed equally to this work

Specialty section: This article was submitted to

Infectious Diseases,

a section of the journal

Frontiers in Microbiology

Received: 03 July 2018 Accepted: 25 February 2019

Published: 15 March 2019

Citation:

De Re V, Tornesello ML,

De Zorzi M, Caggiari L, Pezzuto F, Leone P, Racanelli V, Lauletta G,

Gragnani L, Buonadonna A,

Vaccher E, Zignego AL, Steffan A and

Buonaguro FM (2019) Clinical

Significance of Polymorphisms

in Immune Response Genes

in Hepatitis C-Related Hepatocellular Carcinoma. Front. Microbiol. 10:475.

doi: 10.3389/fmicb.2019.00475

\section{Clinical Significance of Polymorphisms in Immune Response Genes in Hepatitis C-Related Hepatocellular Carcinoma}

Valli De Re ${ }^{1 * t}$, Maria Lina Tornesello ${ }^{2 t}$, Mariangela De Zorzi', Laura Caggiari', Francesca Pezzuto ${ }^{2}$, Patrizia Leone ${ }^{3}$, Vito Racanelli ${ }^{3}$, Gianfranco Lauletta ${ }^{3}$, Laura Gragnani ${ }^{4}$, Angela Buonadonna ${ }^{1}$, Emanuela Vaccher ${ }^{1}$, Anna Linda Zignego ${ }^{4}$, Agostino Steffan ${ }^{1}$ and Franco M. Buonaguro ${ }^{2}$

${ }^{1}$ Centro di Riferimento Oncologico, Cancer Institute, Aviano, Italy, ${ }^{2}$ Istituto Nazionale Tumori IRCCS "Fondazione G. Pascale," Naples, Italy, ${ }^{3}$ Department of Biomedical Sciences and Human Oncology, Section of Internal Medicine, University of Bari "Aldo Moro", Bari, Italy, ${ }^{4}$ Department of Experimental and Clinical Medicine and Department of Oncology, Interdepartmental Hepatology Center MASVE, Azienda Ospedaliero-Universitaria Careggi (AOUC), Florence, Italy

Background and Aims: Polymorphisms in the immune response genes can contribute to clearance of hepatitis $\mathrm{C}$ virus ( $\mathrm{HCV}$ ) infection but also mediate liver inflammation and cancer pathogenesis. This study aimed to investigate the association of polymorphisms in PD-1 (PDCD1), IFNL3 (IL28B), and TLR2 immune related genes in chronic HCV patients with different hepatic and lymphoproliferative HCV-related diseases.

Methods: Selected PDCD1, IFNL3, and TLR2 genes were tested by molecular approaches in $450 \mathrm{HCV}$-positive patients with increasing severity of underlying liver diseases [including chronic infection $(\mathrm{CHC})$, cirrhosis and hepatocellular carcinoma $(\mathrm{HCC})$ ], in $238 \mathrm{HCV}$-positive patients with lymphoproliferative diseases [such as cryoglobulinemia and non-Hodgkin lymphoma (NHL)] and in 94 blood donors (BD).

Results: While the rs12979860 IFNL3 T allele was found a good marker associated with HCV-outcome together with the rs111200466 TLR2 del variant, the rs10204525 PD1.6 A allele was found to have an insignificant role in patients with HCV-related hepatic disorders. Though in Asian patients the combination of IFNL3 and PD-1.6 markers better define the HCV-related outcomes, in our series of Caucasian patients the PD-1.6 A-allele variant was observed very rarely.

Conclusion: Differences in the incidence of HCV-related HCC and clinical response between Asians and Europeans may be partially due to the distribution of PD-1.6 genotype that we found divergent between these two populations. On the other hand, we confirmed in this study that the polymorphic variants within IFNL3 and TLR2 immune response genes are significantly associated with HCV-related disease progression in our cohort of Italian patients.

Keywords: hepatitis virus C, hepatocellular carcinoma, cirrhosis, lymphoproliferative disorders, gene polymorphism, PD-1, IFNL3, TLR2 


\section{INTRODUCTION}

Hepatocellular carcinoma is the primary malignancy of the liver that often occurs in the setting of underlying chronic liver disease, mostly $\mathrm{HBV}$ and/or $\mathrm{C}$ virus infection ( $\mathrm{HBV}$ and $\mathrm{HCV}$, respectively), alcoholic liver disease, and non-alcoholic fatty liver disease. In the last years the incidence rate of HCC has increased in the European and American populations (Ryerson et al., 2016), mostly related to the increase of $\mathrm{HCV}$ infection acquired before the availability of the serologic test.

Curative treatment options for HCC are local resection, radioembolization and multikinase inhibitors. Available options in patients with unresectable HCC are liver transplantation, percutaneous ethanol injection, radiofrequency ablation, and transcatheter arterial chemoembolization (Hernaez and El-Serag, 2018).

Unfortunately, most patients have locally advanced or metastatic HCC at diagnosis and are not eligible for either liver resection or transplantation. In these cases, despite the attempt to improve the OS of patients by chemotherapy, radioembolization, and multikinase inhibitor sorafenib, the OS remains poor (Llovet et al., 2008). The role of tumor-infiltrating leukocytes in mediating cancer progression and efficacy of immunotherapy in other malignancies, like melanoma, are now well recognized. Thus, although liver represents an "immune privileged" organ, immunotherapy now quickly evolves as a treatment option for HCC (Prieto et al., 2015). Based on programmed cell death 1 (PD1) and PD-L ligands checkpoint blockade, the immunotherapy for HCC has shown encouraging results in phase I/II trials of Nivolumab (Checkmate 040 trial) (El-Khoueiry et al., 2017).

The PD-1/PD-L pathway has been demonstrated to be engaged in the inhibition of activated T-cells with PD-1 upregulated in exhausted CD8 T-cells, a mechanism involved in hepatic viral persistence. The PD-1 expression has been shown to associate with the development of HBV-related liver diseases and the prognosis of HCC patients (Zhang et al., 2010; Li et al., 2013; Li et al., 2016). A recent proteomic study analyzing HCC cancer-immune landscape across tumor, non-tumor, and peripheral blood cells demonstrated the existence of a cancerimmune gradient which become progressively suppressive from the non-tumor to the tumor microenvironment (Chew et al., 2017). Specifically, authors have demonstrated the importance of the immunosuppressive action caused by exhausted tumorinfiltrating memory CD8 + T cells expressing high levels of PD-1, that allows immune evasion by the virus and cancer cells (Blank et al., 2005; Park et al., 2015). The increase number of exhausted PD-1+ T-cells was significantly higher in HBV-related vs. nonviral-associated HCC, and much more increased during the HCC progression stage (stage 1 vs. stage $\geq 2$ ) (Chew et al., 2017). PD1 was also found significantly up-regulated in CD8+ cytotoxic

Abbreviations: BD, blood donors; 95\% CI, 95\% confidence interval; CHC, chronic infection; del, deletion; HBV, chronic hepatitis B; HCC, hepatocellular carcinoma; $\mathrm{HCV}$, hepatitis $\mathrm{C}$ virus; ins, insertion; INFL, interferon lambda; MAF, minor allele frequency; MC, mixed cryoglobulinemia; NHL, non-Hodgkin lymphoma; OR, odds ratio; OS, overall survival; PCR, polymerase chain reaction; PD-1, programmed cell death protein 1; SNP, single nucleotide polymorphisms; TLR, toll like receptor; UTR, untranslated region.
T-cells in patients with chronic $\mathrm{HCV}$-infection compared to either HCV-negative subjects or patients with spontaneous HCV resolution (Golden-Mason et al., 2007). In vitro blockade of PD1 has been shown to restore the functional competence of the HCV-specific T-cells (Golden-Mason et al., 2007).

Two SNP on the chromosome 2 within the PDCD1 gene, the rs36084323 G/A (PD-1.1) located -606 base pairs upstream the promoter region at position 242801596 and the rs10204525 G/A (PD-1.6) located at +8669 base pairs in the $3^{\prime}$ UTR at the position 241850169, have been found to be significantly associated with the risk to develop HBV-related cirrhosis and HCC among a Chinese Han population (Zhang et al., 2010; Li et al., 2013; Peng et al., 2015). The mechanisms underlying this association are likely due to the rs $36084323 \mathrm{G}$ allele, positioned in a putative binding site for the UCE-2 transcription regulators, causing the increased expression of PD-1 (Sasaki et al., 2014), and the rs10204525 A allele, disrupting the binding sequence for miR-4717 inhibitor within the $3^{\prime}$ UTR of PD-1 mRNA, which drives increased PD-1 expression (Zhang et al., 2015). In fact, the miRNA-4717 was demonstrated to affect the luciferase activity in a dose-dependent manner in cells transfected with a recombinant vector expressing the luciferase reporter gene under the transcription control of the PD-1 promoter containing the rs10204525 G polymorphic variant (Zhang et al., 2015).

Hepatitis $\mathrm{C}$ virus leads to chronic hepatitis ( $\mathrm{CHC}$ ) and is a major cause of liver cirrhosis and HCC. HCV is also a lymphotropic virus that triggers $\mathrm{B}$-cells and promotes favorable conditions for B lymphocyte proliferation, including the autoimmune condition MC and B-cell non-Hodgkin lymphoma (B-NHL) (De Re et al., 2007; Sansonno et al., 2007).

By exploring the relationship between innate immunity and HCV-related disorders we found that the IFNL3 C rs12979860 and TLR2 -196-174 ins polymorphisms, both associated with interferon-treatment response and spontaneous HCV-clearance as well as with lower HCV viral load, are associated with a decreased risk of HCV-related diseases and delay the occurrence of cirrhosis and HCC (De Re et al., 2016).

In the present study, we simultaneously analyzed the distribution of polymorphic variants in the PD-1, IFNL3, and TLR2 immune-related genes among Italian patients affected by HCV-related CHC, cirrhosis and HCC $(n=450)$ and we compared the genotype and allele frequencies with those obtained in patients affected by HCV-related lymphoproliferative diseases, such as MC and NHL, $(n=238)$ and in healthy $\mathrm{BD}(n=94)$.

\section{PATIENTS AND METHODS}

\section{Study Design}

A total of $148 \mathrm{HCV}$-infected patients with $\mathrm{CHC}$ without cirrhosis or HCC (48.3\% male; median age 57.1 years), 113 patients with $\mathrm{HCV}$-associated cirrhosis (65.4\% males; median age 64.5 years), 189 patients with $\mathrm{HCV}$-associated HCC (73.6\% male; median age 68.9 years), $238 \mathrm{HCV}$-infected patients with lymphoproliferative disorders (130 MC, 29.1\% male, median age 68.0 and $108 \mathrm{NHL}$, $47.5 \%$ male, median age 66.5 years), and 94 healthy BD $(89.6 \%$ 
TABLE 1 | Clinical characteristics and PD-1.6 genotype of 688 HCV-positive patients and 94 HCV-negative BD.

\begin{tabular}{|c|c|c|c|c|c|c|c|c|c|c|}
\hline & \multirow[b]{2}{*}{$n$} & \multirow[b]{2}{*}{ Age } & \multicolumn{8}{|c|}{ PD-1.6 A > G (rs10204525) } \\
\hline & & & Male (\%) & & MAF* & G/G (\%) & A/G (\%) & A/A (\%) & Viral load $^{\# \circ}$ & HCV genotype $^{\circ}(\%)$ \\
\hline \multicolumn{11}{|c|}{ Control subjects } \\
\hline $\mathrm{BD}$ & 94 & $42.46 \pm 10.1$ & $43(89.6)$ & 94 & 0.10 & $76(80.9)$ & $18(19.1)$ & 0 & - & - \\
\hline \multicolumn{11}{|c|}{ HCV infected patients with liver diseases } \\
\hline $\mathrm{CHC}$ & 148 & $57.12 \pm 14.1$ & $56(48.3)$ & 450 & 0.09 & $125(84.5)$ & $20(13.5)$ & $3(2.0)$ & $2.32 \pm 3.9$ & $51 / 82(55.6)$ \\
\hline Cirrhosis & 113 & $64.46 \pm 11.1$ & $70(65.4)$ & & 0.09 & $93(82.3)$ & $19(16.8)$ & $1(0.9)$ & $2.71 \pm 4.4$ & $15 / 27(62.2)$ \\
\hline $\mathrm{HCC}$ & 189 & $68.86 \pm 8.6$ & $103(73.6)$ & & 0.09 & $154(81.5)$ & $34(18.0)$ & $1(0.5)$ & $1.94 \pm 1.9$ & $3 / 3(100)$ \\
\hline \multicolumn{11}{|c|}{ HCV infected patients with lymphoproliferative diseases } \\
\hline $\mathrm{MC}$ & 130 & $68.03 \pm 9.9$ & $32(29.1)$ & 238 & 0.11 & $103(79.2)$ & $26(20.0)$ & $1(0.8)$ & $3.32 \pm 4.4$ & 40/55 (72.7) \\
\hline $\mathrm{NHL}$ & 108 & $66.53 \pm 15.1$ & $47(47.5)$ & & 0.10 & $87(80.6)$ & $20(18.5)$ & $1(0.9)$ & $3.13 \pm 4.2$ & $7 / 11(63.6)$ \\
\hline Total & 782 & $65.9 \pm 13.7$ & $351(44.9)$ & 782 & 0.10 & $638(81.6)$ & $137(17.5)$ & $7(0.9)$ & $2.52 \pm 3.9$ & $116 / 178(65.2)$ \\
\hline
\end{tabular}

BD, blood donors; CHC, chronic hepatitis C; HCC, hepatocellular carcinoma; MC, mixed cryoglobulinemia; NHL, non-Hodgkin lymphoma; \pm , standard deviation; *MAF, minor allele frequency, corresponding to A allele; " viral load, mean in million; ' data of viral load and of HCV genotype type 1 were determined in 178 cases.

male; median age 42.5 years) were included in this study. Some of the individuals recruited for the study are part of a previous study [18]. Cases added as new are: BD $n=94, \mathrm{CHC} n=76$, cirrhosis $=13, \mathrm{HCC}=102, \mathrm{MC}=130, \mathrm{NHL}=12$. Demographic characteristics of the enrolled patients as well as HCV genotype and viral load were summarized in Table 1. Patients with CHC and healthy BD have a lower mean age. Female gender was more frequent among patients with MC.

The diagnosis of chronic HCV infection was based on anti-HCV antibodies, elevated ALT serum levels and HCV RNA positivity for at least 6 months. The diagnosis of HCC was based on the standard criteria listed in the European Association for the Study of the Liver (EASL) that incorporate both invasive and non-invasive measures. Non-invasive criteria include two imaging techniques, both demonstrating a focal lesion $>2 \mathrm{~cm}$ in diameter with features of arterial hypervascularization. Detection and immunochemical characterization of cryoglobulins were performed according to the consensus protocol proposed by the "Associazione Italiana per la Lotta alle Crioglobulinemie." The diagnosis of NHL in the course of HCV infection has been histopathologically confirmed based on the WHO classification.

The study is in accordance with the principles of the Helsinki Declaration and all subjects provided written informed consent. The study was approved by institutional review boards and independent ethics committees since this was a multicenter study. Particularly, the study of HCC cases was approved by the ethical committee EUDRACT (No. 2010-023602-12), Comitato Etico Indipendente of the Azienda Ospedaliero-Universitaria "Consorziale Policlinico" di Bari, the scientific board and the ethics committee of the Istituto Nazionale Tumori "Fond Pascale"; the institutional review board code SPE 14.084_AOUC; comitato Etico Area Vasta Centro AOU Careggi, Firenze.

The HCV antibody test was performed by an enzyme immunoassay (III-generation EIA) against HCV-core and HCVnon-structural antigens. The HCV viral load (RNA UI/mL) was assessed by branched DNA technology (Chiron, Emeryville, CA, United States) in serum samples of 201 patients at the time of diagnosis of the HCV-related disorder. HCV genotype was determined by a commercial, certified, diagnostic test (Versant HCV Genotype 2.0, Siemens Healthcare Diagnostics, Deerfield, IL, United States).

\section{Genotyping of PD-1, IFNL3, and TLR2 Polymorphisms}

We collected $2 \mathrm{~mL}$ of whole blood from each patient and cryopreserved at $-20^{\circ} \mathrm{C}$ until use. Total genomic DNA was extracted from peripheral blood using Qiagen DNAeasy Kit (QIAGEN, Grand Island, NY, United States). We analyzed four polymorphisms within the PD.1, IFNL3, and TLR2 genes, previously described as genetic factors involved in the immune response and hepatic disease progression (Zhang et al., 2010; Park et al., 2015; Li et al., 2016; Asian liver center, 2018; CDC, 2018). They include 3 single-nucleotide changes at positions 606 G/A (rs36084323, PD-1.1) (Xiao et al., 2015) and +8669 G/A (rs10204525, PD-1.6) within the PD-1 gene (Xiao et al., 2015), at position $+1825 \mathrm{C} / \mathrm{T}$ (rs12979860) in the IFNL3 gene (De Re et al., 2016) and a 22-bp nucleotide del/ins from the position -196 to -174 (rs111200466) in the untranslated $5^{\prime}$-region of TLR2 gene (De Re et al., 2016).

Oligonucleotides used for genotyping were listed in Supplementary Table S1. Particularly, PD-1.1 and PD-1.6 were amplified as described by Zhang et al. (2010) by using PCR and products subjected to automated bidirectional direct sequencing analysis (Eurofins Genomics $\mathrm{GmbH}$, Ebersberg, Germany). Briefly, PCR reactions were performed in $50 \mu \mathrm{L}$ reaction mixture containing 30-300 ng of genomic DNA, 10 pmol of each primer, 1.25 Unit of Hot Master Taq DNA Polymerase (5 Prime GmbH, Hamburg, Germany) and $25 \mu \mathrm{L}$ of PreMixJ (MasterAmp ${ }^{\text {TM }}$ PCR, Epicentre, Madison, WI, United States). DNA was amplified in Sure Cycler 8800 thermal cycler (Agilent Technologies, SantaClara, CA, United States) starting with an initial denaturation at $94^{\circ} \mathrm{C}$ for $3 \mathrm{~min}$, followed by 30 amplification cycles of denaturation at $94^{\circ} \mathrm{C}$ for $30 \mathrm{~s}$, annealing at $65^{\circ} \mathrm{C}$ for $30 \mathrm{~s}$, elongation at $72^{\circ} \mathrm{C}$ for 1 and $10 \mathrm{~min}$ 
final elongation at $72^{\circ} \mathrm{C}$. PCR amplification generated a fragment of 730 and $490 \mathrm{bp}$ for the PD-1.1 and PD-1.6, respectively.

IFNL3 genotyping was performed using a specific custom TaqMan SNP-genotyping Assay (SNP rs12979860; Applied Biosystem, Foster City, CA, United States) on a 7900HT Fast Real-Time PCR system (Applied Biosystem, Foster City, CA, United States) (De Re et al., 2016). Determination of TLR2 polymorphism was performed by allele-specific PCR method. Fragments of different length (264 and $286 \mathrm{bp}$ ), depending on the presence or absence of the del mutation were visualized by electrophoresis on a $3.5 \%$ agarose gel stained with ethidium bromide (Supplementary Figure S1). Amplicon sequencing was used to validate the genotyping techniques.

\section{Statistical Analysis}

Specific tests including Fisher's exact test and one or two-way analysis of variance were used to compare allele and genotype frequency of PD-1, TLR2, and IFNL3 polymorphisms between patient groups with different pathologies and control subjects. Multivariate logistic regression analysis was performed with diagnosis as a dependent variable and independent variables, including age, gender ( 0 female; 1 male), and each genotype was also considered. $P$-value, OR and $95 \%$ CIs were calculated. Genotypes of each polymorphism were assessed according to dominant ( 0 wild-type homozygote; 1 heterozygote and variant homozygote), recessive (0 wild-type homozygote and heterozygote; 1 variant homozygote) and additive genetic models. Statistical power calculation was performed by using OSSE online tool ${ }^{1}$. Statistical analyses were performed using GraphPad Prism v6 and SNPStats. $P$ value $<0.05$ was considered statistically significant.

\section{RESULTS}

\section{Genotype Frequencies}

The genotype and allele frequencies of PD-1.6 in HCV-related cases and healthy $\mathrm{BD}$ are listed in Table 1. Male gender was predominant in our cohort of BD (89.6\%), due to psychological, cultural, and social reasons. The analysis of PD-1.6 genotype distributions among $\mathrm{HCV}$-related cases, compared to that of $\mathrm{BD}$ showed no significant association with the risk of development of liver diseases or lymphoproliferative disorders.

The A-allele MAF PD-1.6 was 0.09 in patients with liver diseases, 0.10 in patients with lymphoproliferative disorders and 0.10 in $\mathrm{BD}$. The frequency of PD-1.6 A/A genotype ranged between 0.5 and $2 \%$ in $\mathrm{HCV}$-related cases; $0.8-0.9 \%$ in lymphoproliferative disorders and the allele $\mathrm{A}$, and thus the genotype $\mathrm{A} / \mathrm{A}$, was not found among $\mathrm{BD}$ subjects. Differences in allele frequencies and genotype distribution between HCVrelated diseases and $\mathrm{BD}$ were not statistically significant. By comparing the distribution of PD-1.6 alleles among all HCVrelated liver diseases (CHC, cirrhosis, and HCC) with HCVrelated lymphoproliferative disorders (MC, NHL) a significantly higher frequency of A allele was found in the latter group (83/817

${ }^{1}$ http://osse.bii.a-star.edu.sg/calculation2.php and 50/426, respectively, $p=0.018$ ). However, no statistically significant difference was observed by comparing the A allele distribution in HCV-related liver diseases or in HCV-related lymphoproliferative disorders with that determined in the $\mathrm{BD}$ group. Age and gender of $\mathrm{BD}$ did not affect the result of the study: chi-squared test for trend among individuals with $<40$; $<50$, and $\geq 50$ years old was $p=0.74,0.22$, and 0.62 for PD1, IFNL3, and TLR2, respectively; chi-square test for gender (female vs. male) was $p=0.67,0.83$, and 0.92 and for PD-1, IFNL3, and TLR2, respectively. The allele frequency and genotype distribution were also found independent of HCV viral load and HCV genotype (Table 1).

The PD-1.1 polymorphism was analyzed in 109 HCC cases and consistently with the allele frequency distribution in the Caucasian population all samples were found G/G homozygous for such polymorphism (data not shown).

The analysis of IFNL3 rs12979860 polymorphism was shown in Table 2. There was an increase of $\mathrm{T}$ allele frequency, showing an additive genotype trend, in patients with liver diseases, particularly $\mathrm{CHC}(\mathrm{OR}=1.57 ; 95 \% \mathrm{CI}, 1.06-2.31)$, cirrhosis $(\mathrm{OR}=2.10 ; 95 \% \mathrm{CI}, 1.40-3.16)$, and HCC $(\mathrm{OR}=1.79 ; 95 \%$ CI, 1.21-2.64) compared to BD controls. This analysis had 78\% power to detect differences in IFNL3 C/T allele distribution.

The frequency of IFNL3 T allele was also higher in patients with hepatic diseases compared to the lymphoproliferative diseases (MAF 0.44 vs. 0.36; OR $=1.77$, 95\%CI 1.40-2.25, $p<0.0001)$. In particular, patients with more advanced HCVrelated liver diseases (i.e., cirrhosis and HCC) the frequency of IFNL3 T/T homozygous genotype was 1.4-fold higher than in MC and NHL, and 3.3 higher than in BD (Figure 1 and Table 2). The IFNL3 T/T genotype was also 2.3-fold higher in MC and NHL patients compared to BD (Figure 1).

The distribution of TLR2 ins/del genotypes is shown in Table 2. The frequencies of these alleles in the HCV-related groups did not indicate any significant association, with the exception of the -196 to -174 del that was significantly more represented among HCC patients compared to BD controls (del vs. ins $\mathrm{OR}=1.97 ; 95 \% \mathrm{CI}, 1.17-3.31 ; p=0.01$ ). Moreover, we found a statistically significant difference in the frequency of del allele in HCV-related patients with MC lymphoproliferative disease compared to controls $(\mathrm{OR}=1.71$; 95\% CI 1.00-2.91; $p=0.05$ ).

Despite the limited power to detect the effect of TLR2 polymorphism, due to the low MAF, a statistically significant linear trend has been observed for TLR2 del/del genotype (Chisquare $=9.94, p=0.0016)$ among the HCV-related groups.

\section{Epistatic Interaction Between IFNL3 and TLR2}

The above results indicated that only the polymorphic variations in IFNL3 and TLR2 genes were associated with susceptibility to HCV-related diseases in our series (Tables 1, 2). A general linear regression model was used to identify multiloci genotypes associated with different HCV-related diseases. For the analysis, IFNL3-TLR2 genotypes from HCV-related liver and HCV lymphoproliferative diseases were divided into 4 groups, coded 
TABLE 2 | IFNL3 and TLR2 genotypes among 688 HCV-positive cases and 94 HCV-negative BD.

\begin{tabular}{|c|c|c|c|c|c|c|c|c|c|c|c|}
\hline & \multirow[b]{2}{*}{$n$} & \multicolumn{4}{|c|}{ IFNL3 C > T (rs12979860) } & \multirow[b]{2}{*}{ OR (95\%Cl) } & \multicolumn{4}{|c|}{ TLR-2 ins/del rs111200466) } & \multirow[b]{2}{*}{$\begin{array}{c}\text { OR } \\
(95 \% \mathrm{Cl})^{\dagger}\end{array}$} \\
\hline & & $\mathrm{C} / \mathrm{C}(\%)$ & $\mathrm{C} / \mathrm{T}(\%)$ & $\mathrm{T} / \mathrm{T}(\%)$ & MAF* & & Ins/Ins (\%) & Ins/Del (\%) & Del/Del (\%) & MAF* $^{*}$ & \\
\hline \multicolumn{12}{|c|}{ Control subjects } \\
\hline BD & 94 & $42(44.7)$ & $47(50.0)$ & $5(5.3)$ & 0.30 & Reference & $72(76.4)$ & $21(22.3)$ & $1(1.4)$ & 0.12 & Reference \\
\hline \multicolumn{12}{|c|}{ HCV infected patients with liver diseases } \\
\hline $\mathrm{CHC}$ & 148 & $44(29.7)$ & $88(59.4)$ & $16(10.8)$ & 0.41 & $\begin{array}{c}1.57(1.1-2.3) \\
p=0.02\end{array}$ & $101(68.2)$ & $41(27.0)$ & $6(4.7)$ & 0.16 & \\
\hline Cirrhosis & 113 & $26(23.0)$ & $66(58.4)$ & $21(18.6)$ & 0.48 & $\begin{array}{c}2.10(1.4-3.2) \\
p<0.001\end{array}$ & 82 (72.6) & $26(23.0)$ & $5(4.4)$ & 0.16 & \\
\hline $\mathrm{HCC}$ & 144 & $42(29.2)$ & $78(54.2)$ & $24(16.7)$ & 0.43 & $\begin{array}{c}1.79(1.2-2.6) \\
p=0.003\end{array}$ & $97(67.4)$ & $32(22.2)$ & $15(10.4)$ & 0.21 & $\begin{array}{c}1.97(1.2-3.3) \\
p=0.011\end{array}$ \\
\hline \multicolumn{12}{|c|}{ HCV infected patients with lymphoproliferative diseases } \\
\hline $\mathrm{MC}$ & 130 & $55(42.3)$ & $59(45.4)$ & $16(12.3)$ & 0.35 & & $86(66.2)$ & $38(29.2)$ & $6(4.6)$ & 0.19 & $\begin{array}{c}1.71(1.0-2.9) \\
p=0.050\end{array}$ \\
\hline $\mathrm{NHL}$ & 108 & $41(38.0)$ & $54(50.0)$ & $13(12.0)$ & 0.37 & & 83 (76.9) & $24(22.2)$ & $1(0.9)$ & 0.12 & \\
\hline Total & 737 & 250 (33.9) & 392 (53.2) & 95 (12.9) & 0.40 & & $521(70.7)$ & $182(24.7)$ & $34(4.6)$ & 0.16 & \\
\hline
\end{tabular}

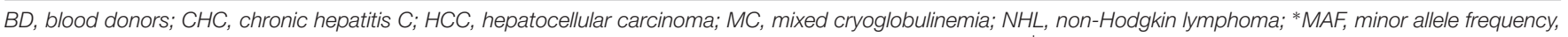
corresponding to deletion (del) in TLR2 and T-allele in IFNL3; OR, odds ratio; 95\% CI, 95\% confidence interval; ${ }^{\dagger}$ only significant results have been reported.

as InsC, InsT DelC, DelT and their frequencies were compared to those obtained in $\mathrm{BD}$ and lymphoproliferative vs. liver diseases (Table 3). Wild-type TLR2-ins- IFNL3-C was the most frequent group (Table 3). Some multilocus genotypes, i.e., ins-T and del$\mathrm{T}$ distinguished patients with liver diseases compared to $\mathrm{BD}$ (OR $=1.68 ; 95 \%$ CI 1.06-2.65, $p=0.028)$ and lymphoproliferative vs. HCV-related liver disorders $[(\mathrm{OR}=0.72 ; 95 \%$ CI $0.56-$ $0.94), p=0.014$ and (OR $=0.43$; 95\% CI 0.22-0.85), $p=0.016$, respectively], indicating that these multi loci genes play a significant role in the development of liver diseases among HCVpositive subjects.

Comparison of IFNL3 T-allele distribution between groups of patients affected by different HCV-related liver diseases and healthy $\mathrm{BD}$ underlined the role of such polymorphic variant as dominant key factor for the progression of cirrhosis to the most advanced liver diseases in our series (Table 3).

\section{PD-1.6 and IFNL3 MAF Frequencies in Different Countries}

Surveys of HBV infection and the rate of HCC in different geographic regions showed a great disparity between Asian and other populations. In fact, the incidence of liver cancer is about nine-fold higher in Asians compared to white Americans suggesting that genetic polymorphisms and environmental risk factors may be responsible for such divergences (Asian liver center, 2018; CDC, 2018). Therefore, we compared the frequencies of PD-1.6 and IFNL3 polymorphisms in different countries reported in the NCBI database ${ }^{2}$ and the frequencies found in our series (Table 4). A significant difference in allele distribution among Asian and Italian population was observed both for the PD-1.6 (MAF 0.66 vs. 0.10 ) and IFNL3 (0.08 vs. 0.31) polymorphisms as shown in Table 4.

\footnotetext{
${ }^{2}$ http://www.ncbi.nlm.nih.gov/SNP/
}

\section{DISCUSSION}

Previous studies have demonstrated that elevated expression of PD-1 in lymphocytes within the liver, especially exhausted $\mathrm{T}$ cells and Tregs, are closely associated with a dysfunction of the immune response in chronic HBV infection and HBVrelated HCC (Boni et al., 2007; Fisicaro et al., 2010; Hsu et al., 2010; Wang et al., 2011). Moreover, it has been reported that PD1.1 and PD-1.6 polymorphisms combined with chronic HBV infection contribute to the development of HCC in a Chinese population (Li et al., 2013) and polymorphisms concur in the development of several tumor types and autoimmune disease pathogenesis (Momin et al., 2009; Liu et al., 2011; Tahoori et al., 2011; Li et al., 2013; Tang et al., 2017; Tejeda et al., 2017; Salmaninejad et al., 2018).

Studies focusing on CHC, by Penna et al. (2007) and Radziewicz et al. (2007) have shown that up-regulation of PD-1 affects HCV-specific CD8+ $\mathrm{T}$ cell function in the intrahepatic compartment in patient with chronic $\mathrm{HCV}$ infection. Blockade of the PD-1/PD-L1 interaction was shown to improve the expansion ability and IFN- $\gamma$ secretion from HCV-specific CD8+ T cells (Moreno-Cubero and Larrubia, 2016) and control HCV replication in a chimpanzee model of $\mathrm{CHC}$, although the efficacy was noted only in those animals with a critical threshold of pre-existing HCV-specific CD8+ T cells (Fuller et al., 2013). Additional studies showed that PD-1 is also critical in the persistence of chronic viral infections in mice (Barber et al., 2006) and in the progression of acquired immunodeficiency syndrome in humans (Day et al., 2006).

In our series we found a slight but not statistically significant increase of the PD-1.6 A/A genotype in the whole group of patients with $\mathrm{HCV}$-related disorders compared to the control group of $\mathrm{BD}$. However, the frequency of PD-1.6 A/A genotype is 


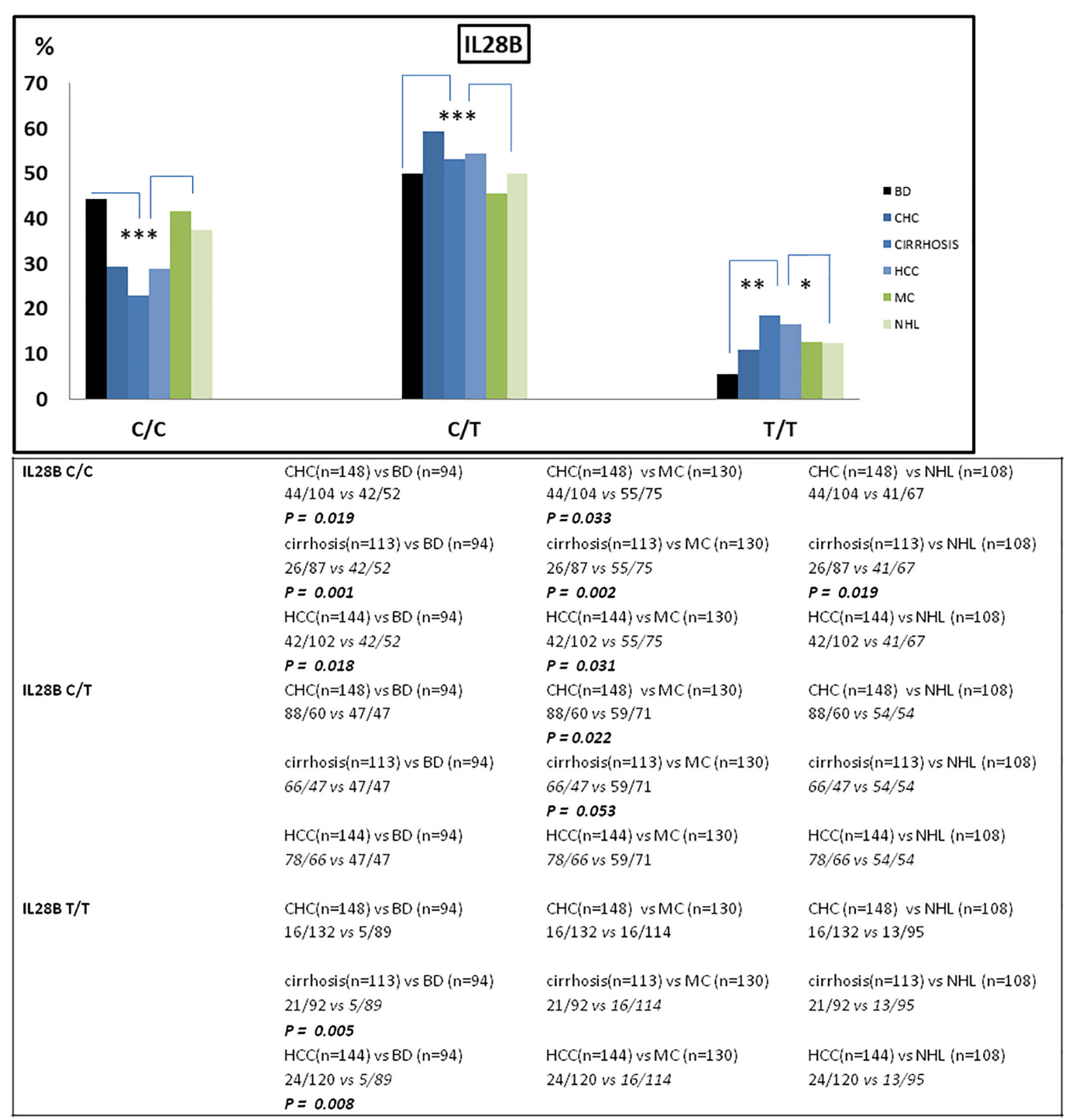

FIGURE 1 | Frequency of IFNL3 genotype distribution among patients stratified on the basis of their HCV-related disease $(n=643)$ and blood donors (BD) $(n=94)$. The frequency of IFNL3 T allele was found increased in HCV-associated liver diseases (CHC, cirrhosis, and HCC) compared to BD and to lymphoproliferative disorders (MC and $\mathrm{NHL})$. BD, blood donors (controls); $\mathrm{CHC}$, chronic hepatitis $\mathrm{C}$; HCC, hepatocellular carcinoma; MC, mixed cryoglobulinemia; NHL, non-Hodgkin lymphoma. ${ }^{*} p<0.05 ;{ }^{* *} p<0.001 ;$ and ${ }^{* * *} p<0.005$.

very limited, ranging from 0 to $2 \%$ (Table 1 ) resulting in a allele-A MAF of 0.10 (Table 4), while this unfavorable A/A genotype is the most common genotype (52.6\%) in Asian population, with a allele-A MAF of about 0.66 (Table 4; Tang et al., 2017; Tejeda et al., 2017).

There are remarkable dissimilarities in the distribution of PD-1.6 polymorphic variants and their association with HCC between the Asian population and our Italian cohort. Since each of these studies comprised almost 1000 cases we are incline to think that differences in the PD-1.6 genotype distribution are consistent and reflect the genetic heterogeneity among various populations. On the contrary to Asian population, in our series we found a very low frequency of PD-1.6 A-allele variant (MAF 0.10 , Table 4), thus it is hard to think that this mutation has a strong role in HCC in our population. Additionally, it is well known that persistent $\mathrm{HBV}$ infection were more likely to be associated with HCC in Asian population, while HCV infection had a higher prevalence among the Caucasian population (Ahmad et al., 2018; Falla et al., 2018). Further studies are needed to determine the distribution of PD-1.6 variants in different geographic regions and to explore their casual role in HCV-related diseases susceptibility worldwide.

Since the PD-1/PD-L1 blockade has proven to be an efficient treatment for HCC (Kudo, 2016), the lack of parallel changes in the frequency of PD-1.6 A allele in HCV-related HCC patients and controls in our series excludes a simple direct effect of PD-1.6 variant in the pathogenesis of HCC. However, we cannot exclude the possibility that other polymorphisms in 
TABLE 3 | Comparison of TLR2 and IFNL3 multilocus genotypes frequencies of HCV-related patients with liver $(n=405)$, HCV-related lymphoproliferative diseases $(n=238)$ and blood donors $(n=94)$.

\begin{tabular}{|c|c|c|c|c|c|c|c|c|c|c|c|c|}
\hline IFNL3 & TLR2 & BD & $\mathrm{CHC}$ & Cirrhosis & $\mathrm{HCC}$ & $\begin{array}{l}\text { Hepatic } \\
\text { diseases }\end{array}$ & $\begin{array}{l}\text { Hepatic vs. BD } \\
\text { OR }(95 \% \mathrm{Cl}), \mathrm{p}^{\dagger}\end{array}$ & BD & MC & NHL & Lympho-proliferative & $\begin{array}{l}\text { Lympho-proliferative } \\
\text { vs. hepatic } \\
\text { OR }(95 \% \mathrm{Cl}), \mathrm{p}^{\dagger}\end{array}$ \\
\hline C & Ins & 0.58 & 0.47 & 0.45 & 0.45 & 0.45 & & 0.58 & 0.54 & 0.53 & 0.53 & \\
\hline $\mathrm{T}$ & Ins & 0.29 & 0.36 & 0.39 & 0.35 & 0.37 & $\begin{array}{c}1.68(1.1-2.7) \\
p=0.028\end{array}$ & 0.29 & 0.30 & 0.35 & 0.32 & $0.72(0.6-0.9) p=0.014$ \\
\hline C & Del & 0.10 & 0.11 & 0.10 & 0.13 & 0.11 & & 0.10 & 0.12 & 0.10 & 0.11 & \\
\hline $\mathrm{T}$ & Del & 0.03 & 0.07 & 0.07 & 0.07 & 0.07 & & 0.03 & 0.05 & 0.02 & 0.04 & $0.43(0.2-0.9) p=0.016$ \\
\hline
\end{tabular}

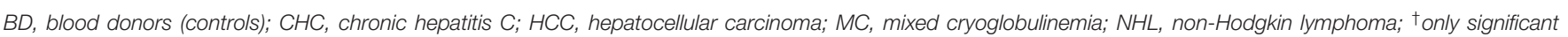
results were reported. OR, odds ratio; $95 \% \mathrm{Cl}$, 95\% confidence interval.

TABLE 4 | Distribution of PD-1.6 allele-A and IFNL3 allele-T frequencies in different populations (available in http://www.ncbi.nlm.nih.gov/SNP/ database) and in our series of HCV-related diseases $(n=710)$.

\begin{tabular}{|c|c|c|c|c|c|c|c|c|}
\hline & MAF & Asian & Nigerian & African & American & European & Italian & HCV* $^{*}$ \\
\hline PD-1.6 & A & 0.66 & 0.50 & 0.39 & 0.39 & 0.12 & $0.10^{\circ}$ & 0.10 \\
\hline IFNL3 & $\mathrm{T}$ & 0.08 & 0.50 & 0.67 & 0.40 & 0.31 & $0.31^{\&}$ & 0.40 \\
\hline
\end{tabular}

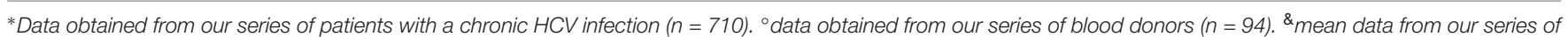
HCV-negative patients ( $n=94$ ); $n=134$ (Taliani et al., 2013); $n=428$ (Falleti et al., 2011).

PD-1 or in other immune-related genes, such as the rs12979860 polymorphism in IFNL3 gene, could be involved in HCVrelated diseases in our Italian population (Ge et al., 2009; Riva et al., 2014; Wack et al., 2015). Alternatively, the discordant correlation between PD-1.6 and HCV-related HCC susceptibility across Asian-European populations could be related to an interaction of the host PD-1.6 gene variant with different environmental factor(s) present in the two populations or HCC development could be related to a different immune check point molecule blockade. Given the important involvement of
PD-1 in autoimmunity and chronic viral infections, further researches are deserved to clarify the role of PD-1 polymorphism in these settings.

Genetic polymorphism of IFNL3 was found strongly associated with spontaneous resolution of $\mathrm{HCV}$ infection and with response to PEGylated interferon-alpha and ribavirin therapy for chronic HCV (Ge et al., 2009; Tanaka et al., 2009; Xiao et al., 2015; Huang et al., 2017). The IFNL3 and PD-1 markers in conjunction have also been reported to influence the susceptibility and outcomes of HCV infection in the Southeast

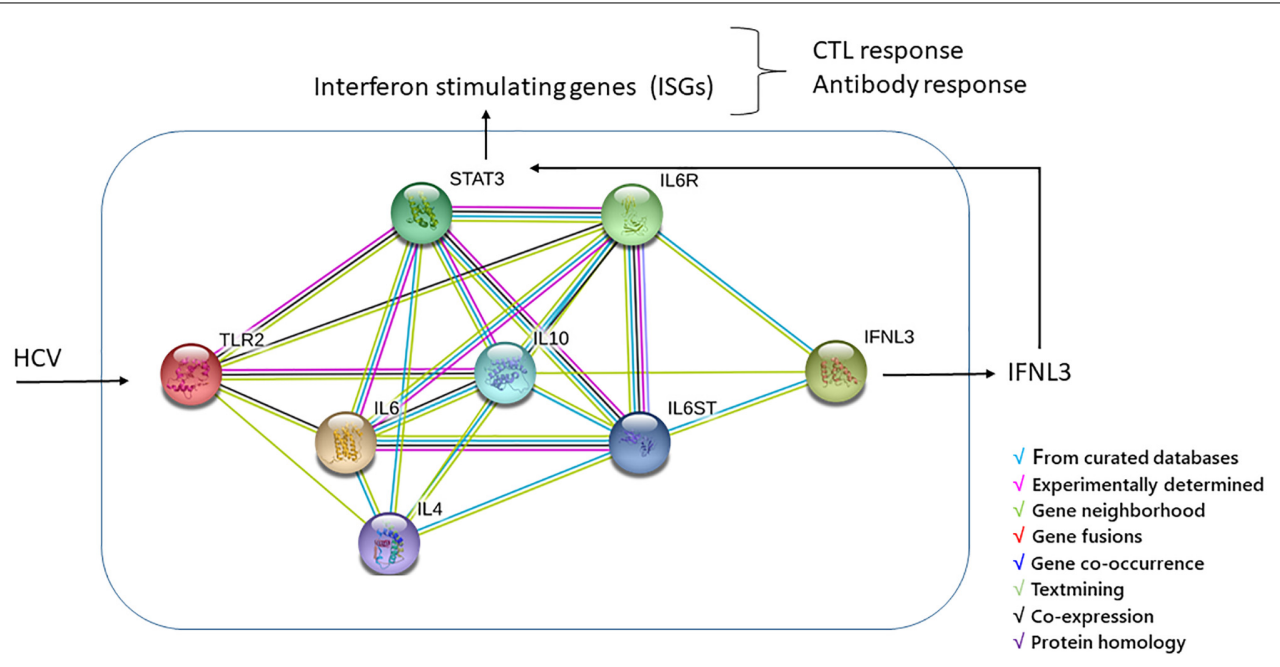

FIGURE 2 | TLR2/IFN- $\lambda 3$ protein-protein interaction was performed by using String software. The interleukin 6 (IL6) and INF- $\alpha$, connecting TLR2 and IFN- $\lambda 3$, can turn on the JAK/STAT pathway, the most important pathway in mediating the inflammatory response to HCV via induction of IFN-stimulated genes (ISGs). The final effect of ISGs results in antigen presentation and a differential expression profile of certain immunomodulatory genes targeting different immune cells and resulting in the polarization of Th1 and Th2 cells, in the modulation of regulatory T-cells and pro-inflammatory cytokines and in the differentiation of dendritic cells (DCs) from monocytes (Egli et al., 2014; Douam et al., 2017; Hemann et al., 2017; Syedbasha and Egli, 2017; Zhou et al., 2018). 
China, suggesting their interactions in the disease outcomes (Xiao et al., 2015). In a previous study we found an association between TLR2 ins/del and IFNL3 polymorphisms with HCVrelated outcome (De Re et al., 2016). In the present study we demonstrated that the multilocus TLR2-ins/ IFNL3 T genotype was a significant factor for development of HCV-related liver diseases (Table 3), and that the impact of rare PD-1.6 variant in Italian population is responsible for the discrepancy between Asian and European results (Table 4). In our series the IFNL3 T variant was confirming to be one of the best markers associated with HCV-related pathogenesis, with a marginal role of TLR2 del variant, while in Asian populations the combined IFNL3 and PD-1.6 polymorphisms were found to better define the HCVrelated outcomes.

Today, we have no data to demonstrate the effect of an interaction between TLR2 and IFNL3 gene products in HCVpositive patients, nonetheless, a functional links between these genes may be indirectly determined using the STRING ${ }^{3}$ software based on genomic associations of genes that are required for a same function. Figure 2 shows the graphical representation of the model of interaction between TLR2 and IFNL3 leading to effect of IFNL3 gene expression on the janus kinase (JAK)/signal transducer and activator of transcription (STAT) (JAK/STAT) pathway. HCV core and NS3 proteins are known to be able to trigger inflammatory pathways via TLR2, which may act, along with TLR1 and TLR6, as a receptor contributing to the activation of the innate immune system and production of interleukin 6 (IL6) and Interferon-alpha (IFN- $\alpha$ ) (Dolganiuc et al., 2004; Chang et al., 2007). In the past before direct-acting antiviral (DAA) treatment IFN- $\alpha$ therapy was largely demonstrated to reduce the risk of HCC and complications associated with cirrhosis in $\mathrm{HCV}$ infected individuals and serum IL- 6 elevation has been correlated with liver disease severity, HCV-RNA titer and the activation of the JAK/STAT pathway (Malaguarnera et al., 1997; Sansone and Bromberg, 2012; Egli et al., 2014; Kong et al., 2016; Hemann et al., 2017; Syedbasha and Egli, 2017; Yakut et al., 2018). IFNL3 signal, producing IFN- $\lambda 3$ molecules, has been demonstrated to inhibit $\mathrm{HCV}$ infection and induce anti-viral response also through the JAK-STAT pathway via induction of IFN-stimulated genes (ISGs) (Malaguarnera et al., 1997; Dolganiuc et al., 2004; Yakut et al., 2018). IFNL3 induces a cell type specific immune response due to the cellular expression of IFN- $\lambda 3$ s receptors in fewer cell types (Egli et al., 2014) and activates the JAK-STAT pathway by a feed-forward fashion with substantial differences in terms of the ISGs gene expression induced by IFN- $\alpha$. Indeed, IFN- $\lambda 3$ showed many antiviral properties but with an overall smaller response than IFN- $\alpha$ causes (Hemann et al., 2017; Syedbasha and Egli, 2017; Zhou et al., 2018). The IFN- $\lambda 3$ effect is mainly associated with antigen presentation and a differential expression profile of certain immunomodulatory genes compared to IFN- $\alpha$ and this suggests a specific functional role for IFN- $\lambda 3$. A critical role of IFN- $\lambda 3 \mathrm{n}$ in the polarization of Th1 and Th2 cells, in the modulation of regulatory T-cells and pro-inflammatory cytokines and in the differentiation of dendritic cells (DCs) have been

\footnotetext{
${ }^{3}$ https://string-db.org/cgi/input.pl?sessionId=7AG0SO9\%20cCRu4\&input\%20_ page_show_search $=$ on
}

well described in several reviews (Egli et al., 2014; Douam et al., 2017; Hemann et al., 2017; Syedbasha and Egli, 2017; Zhou et al., 2018). Of note, during infection with HCV, the expression pattern of many of the ISGs significantly change, most likely due to immunomodulatory effects of HCV proteins and complex inhibitory effects of IFN signaling pathways (Thomas et al., 2012; Egli et al., 2014). In particular, the long-term effects on the Th1/Th2 balance might have implications for the priming of Tand B-cell dependent memory responses, and thus possibly on HCV-related lymphoproliferative malignancy and autoimmune disease prevalence (Egli et al., 2014).

Thus, an indirect interaction between IFNL3 and TLR2 gene products may be suggested from data of literature, but further studies are necessary to confirm the effect of IFNL3 and TLR2 polymorphisms in the prediction of the above reported functional signaling in HCV patients.

This is the first study evaluating the PD1 polymorphisms and the risk of HCV-related disorders in the Italian population. The results should be regarded as descriptive observations and larger studies with more diverse ethnic populations are needed to confirm the association of immune related gene polymorphisms in HCV-related diseases.

In conclusion our study highlighted the importance of geographical difference in the frequencies of PD-1 and IFNL3 genetic polymorphisms in HCV-related diseases particularly in cirrhosis and in HCC susceptibility. Due to the importance of these genes in the immune response to hepatic infection, autoimmune disorders and malignancies as well as their role in the response to new proposed immune check-point treatment for HCC, further studies are needed to better understand the pathogenic role of these genetic variants in HCVrelated diseases.

\section{AUTHOR CONTRIBUTIONS}

VD and MT wrote the manuscript, provided critical discussion in the manuscript preparation, and revised the manuscript. $\mathrm{MD}$ and FP performed the experiments and revised the manuscript. LC, PL, and LG contributed to analyze the data and revise the manuscript. $\mathrm{VR}, \mathrm{LG}, \mathrm{AB}, \mathrm{EV}, \mathrm{AZ}, \mathrm{AS}$, and $\mathrm{FB}$ contributed to collect and analyze the clinical patient's data and revise the manuscript.

\section{FUNDING}

MD and LC fellowships were funded by 5X1000_2010_MdS. FP was the recipient of a research fellowship awarded by FIRE/AISF ONLUS (Fondazione Italiana per la Ricerca in Epatologia) http://www.fondazionefegato.it/.

\section{SUPPLEMENTARY MATERIAL}

The Supplementary Material for this article can be found online at: https://www.frontiersin.org/articles/10.3389/fmicb. 2019.00475/full\#supplementary-material 


\section{REFERENCES}

Ahmad, A. A., Falla, A. M., Duffell, E., Noori, T., Bechini, A., Reintjes, R., et al. (2018). Estimating the scale of chronic hepatitis B virus infection among migrants in EU/EEA countries. BMC Infect. Dis. 18:34. doi: 10.1186/s12879017-2921-8

Asian liver center (2018). Available at: https//liver.standfort.edu.

Barber, D. L., Wherry, E. J., Masopust, D., Zhu, B., Allison, J. P., Sharpe, A. H., et al. (2006). Restoring function in exhausted CD8 T cells during chronic viral infection. Nature 439, 682-687. doi: 10.1038/nature04444

Blank, C., Gajewski, T. F., and Mackensen, A. (2005). Interaction of PD-L1 on tumor cells with PD-1 on tumor-specific $\mathrm{T}$ cells as a mechanism of immune evasion: implications for tumor immunotherapy. Cancer Immunol. Immunother. 54, 307-314. doi: 10.1007/s00262-004-0593-X

Boni, C., Fisicaro, P., Valdatta, C., Amadei, B., Di, V. P., Giuberti, T., et al. (2007). Characterization of hepatitis B virus (HBV)-specific T-cell dysfunction in chronic HBV infection. J. Virol. 81, 4215-4225. doi: 10.1128/JVI.02844-06

CDC (2018). Draft ACIP Hepatitis Recommandations. Atlanta, GA: CDC.

Chang, S., Dolganiuc, A., and Szabo, G. (2007). Toll-like receptors 1 and 6 are involved in TLR2-mediated macrophage activation by hepatitis $\mathrm{C}$ virus core and NS3 proteins. J. Leukoc. Biol. 82, 479-487. doi: 10.1189/jlb.0207128

Chew, V., Lai, L., Pan, L., Lim, C. J., Li, J., Ong, R., et al. (2017). Delineation of an immunosuppressive gradient in hepatocellular carcinoma using highdimensional proteomic and transcriptomic analyses. Proc. Natl. Acad. Sci. U.S.A. 114, E5900-E5909. doi: 10.1073/pnas.1706559114

Day, C. L., Kaufmann, D. E., Kiepiela, P., Brown, J. A., Moodley, E. S., Reddy, S., et al. (2006). PD-1 expression on HIV-specific T cells is associated with T-cell exhaustion and disease progression. Nature 443, 350-354. doi: 10.1038/ nature 05115

De Re, V., Caggiari, L., Simula, M. P., De, V. S., Sansonno, D., and Dolcetti, R. (2007). B-cell lymphomas associated with HCV infection. Gastroenterology 132, 1205-1207. doi: 10.1053/j.gastro.2007.02.009

De Re, V., De, Z. M., Caggiari, L., Lauletta, G., Tornesello, M. L., Fognani, E., et al. (2016). HCV-related liver and lymphoproliferative diseases: association with polymorphisms of IL28B and TLR2. Oncotarget 7, 37487-37497. doi: 10.18632/oncotarget.9303

Dolganiuc, A., Oak, S., Kodys, K., Golenbock, D. T., Finberg, R. W., KurtJones, E., et al. (2004). core and nonstructural 3 proteins trigger toll-like receptor 2-mediated pathways and inflammatory activation. Gastroenterology 127, 1513-1524. doi: 10.1053/j.gastro.2004.08.067

Douam, F., Soto Albrecht, Y. E., Hrebikova, G., Sadimin, E., Davidson, C., Kotenko, S. V., et al. (2017). Type III interferon-mediated signaling is critical for controlling live attenuated yellow fever virus infection in vivo. mBio 8:e819-17. doi: 10.1128/mBio.00819-17

Egli, A., Santer, D. M. O., Shea, D., Tyrrell, D. L., and Houghton, M. (2014). The impact of the interferon-lambda family on the innate and adaptive immune response to viral infections. Emerg. Microbes Infect. 3:e51. doi: 10.1038/emi. 2014.51

El-Khoueiry, A. B., Sangro, B., Yau, T., Crocenzi, T. S., Kudo, M., Hsu, C., et al. (2017). Nivolumab in patients with advanced hepatocellular carcinoma (CheckMate 040): an open-label, non-comparative, phase 1/2 dose escalation and expansion trial. Lancet 389, 2492-2502. doi: 10.1016/S0140-6736(17) 31046-2

Falla, A. M., Ahmad, A. A., Duffell, E., Noori, T., and Veldhuijzen, I. K. (2018). Estimating the scale of chronic hepatitis $\mathrm{C}$ virus infection in the EU/EEA: a focus on migrants from anti-HCV endemic countries. BMC Infect. Dis. 18:42. doi: 10.1186/s12879-017-2908-5

Falleti, E., Bitetto, D., Fabris, C., Cussigh, A., Fornasiere, E., Cmet, S., et al. (2011). Role of interleukin 28B rs $12979860 \mathrm{C} / \mathrm{T}$ polymorphism on the histological outcome of chronic hepatitis C: relationship with gender and viral genotype. J. Clin. Immunol. 31, 891-899. doi: 10.1007/s10875-011-9547-1

Fisicaro, P., Valdatta, C., Massari, M., Loggi, E., Biasini, E., Sacchelli, L., et al. (2010). Antiviral intrahepatic T-cell responses can be restored by blocking programmed death-1 pathway in chronic hepatitis B. Gastroenterology 138, 682-693. doi: 10.1053/j.gastro.2009.09.052

Fuller, M. J., Callendret, B., Zhu, B., Freeman, G. J., Hasselschwert, D. L., Satterfield, W., et al. (2013). Immunotherapy of chronic hepatitis C virus infection with antibodies against programmed cell death-1 (PD-1). Proc. Natl. Acad. Sci. U.S.A. 110, 15001-15006. doi: 10.1073/pnas.1312772110

Ge, D., Fellay, J., Thompson, A. J., Simon, J. S., Shianna, K. V., Urban, T. J., et al. (2009). Genetic variation in IL28B predicts hepatitis C treatment-induced viral clearance. Nature 461, 399-401. doi: 10.1038/nature08309

Golden-Mason, L., Palmer, B., Klarquist, J., Mengshol, J. A., Castelblanco, N., and Rosen, H. R. (2007). Upregulation of PD-1 expression on circulating and intrahepatic hepatitis $\mathrm{C}$ virus-specific CD8+ T cells associated with reversible immune dysfunction. J. Virol. 81, 9249-9258. doi: 10.1128/JVI.00409-07

Hemann, E. A., Gale, M., and Savan, R. (2017). Interferon lambda genetics and biology in regulation of viral control. Front. Immunol. 8:1707. doi: 10.3389/ fimmu.2017.01707

Hernaez, R., and El-Serag, H. B. (2018). How we approach it: treatment options for hepatocellular carcinoma. Am. J. Gastroenterol. 113, 791-794. doi: 10.1038/ s41395-018-0008-9

Hsu, P. N., Yang, T. C., Kao, J. T., Cheng, K. S., Lee, Y. J., Wang, Y. M., et al. (2010). Increased PD-1 and decreased CD28 expression in chronic hepatitis B patients with advanced hepatocellular carcinoma. Liver Int. 30, 1379-1386. doi: 10.1111/j.1478-3231.2010.02323.x

Huang, H., Duggal, P., Thio, C. L., Latanich, R., Goedert, J. J., Mangia, A., et al. (2017). Fine-mapping of genetic loci driving spontaneous clearance of hepatitis C virus infection. Sci. Rep. 20:15843. doi: 10.1038/s41598-017-16011-2

Kong, L., Zhou, Y., Bu, H., Lv, T., Shi, Y., and Yang, J. (2016). Deletion of interleukin-6 in monocytes/macrophages suppresses the initiation of hepatocellular carcinoma in mice. J. Exp. Clin. Cancer Res. 35:131. doi: 10.1186/ s13046-016-0412-1

Kudo, M. (2016). Immune checkpoint blockade in hepatocellular carcinoma: 2017 update. Liver Cancer 6, 1-12. doi: 10.1159/000449342

Li, Z., Li, N., Li, F., Zhou, Z., Sang, J., Chen, Y., et al. (2016). Immune checkpoint proteins PD-1 and TIM-3 are both highly expressed in liver tissues and correlate with their gene polymorphisms in patients with HBV-related hepatocellular carcinoma. Medicine 95:e5749. doi: 10.1097/MD.0000000000005749

Li, Z., Li, N., Zhu, Q., Zhang, G., Han, Q., Zhang, P., et al. (2013). Genetic variations of PD1 and TIM3 are differentially and interactively associated with the development of cirrhosis and HCC in patients with chronic HBV infection. Infect. Genet. Evol. 14, 240-246. doi: 10.1016/j.meegid.2012.12.008

Liu, X., Hu, L. H., Li, Y. R., Chen, F. H., Ning, Y., and Yao, Q. F. (2011). Programmed cell death 1 gene polymorphisms is associated with ankylosing spondylitis in chinese han population. Rheumatol. Int. 31, 209-213. doi: 10.1007/s00296-009-1264-1

Llovet, J. M., Ricci, S., Mazzaferro, V., Hilgard, P., Gane, E., Blanc, J. F., et al. (2008). Sorafenib in advanced hepatocellular carcinoma. N. Engl. J. Med. 359, 378-390. doi: 10.1056/NEJMoa0708857

Malaguarnera, M., Di, F. I., Romeo, M. A., Restuccia, S., Laurino, A., and Trovato, B. A. (1997). Elevation of interleukin 6 levels in patients with chronic hepatitis due to hepatitis C virus. J. Gastroenterol. 32, 211-215. doi: 10.1007/BF02936370

Momin, S., Flores, S., Angel, B. B., Codner, D. E., Carrasco, P. E., and PerezBravo, F. (2009). Interactions between programmed death 1 (PD-1) and cytotoxic T lymphocyte antigen 4 (CTLA-4) gene polymorphisms in type 1 diabetes. Diabetes Res. Clin. Pract. 83, 289-294. doi: 10.1016/j.diabres.2008.12. 003

Moreno-Cubero, E., and Larrubia, J. R. (2016). Specific CD8(+) T cell response immunotherapy for hepatocellular carcinoma and viral hepatitis. World J. Gastroenterol. 22, 6469-6483. doi: 10.3748/wjg.v22.i28.6469

Park, H. J., Park, J. S., Jeong, Y. H., Son, J., Ban, Y. H., Lee, B. H., et al. (2015). $\mathrm{PD}-1$ upregulated on regulatory $\mathrm{T}$ cells during chronic virus infection enhances the suppression of CD8 $+\mathrm{T}$ cell immune response via the interaction with PD-L1 expressed on CD8+ T cells. J. Immunol. 194, 5801-5811. doi: 10.4049/ jimmunol.1401936

Peng, H., Li, Q. L., Hou, S. H., Hu, J., Fan, J. H., and Guo, J. J. (2015). Association of genetic polymorphisms in CD8 $+\mathrm{T}$ cell inhibitory genes and susceptibility to and progression of chronic HBV infection. Infect. Genet. Evol. 36, 467-474. doi: 10.1016/j.meegid.2015.08.018

Penna, A., Pilli, M., Zerbini, A., Orlandini, A., Mezzadri, S., Sacchelli, L., et al. (2007). Dysfunction and functional restoration of HCV-specific CD8 responses in chronic hepatitis C virus infection. Hepatology 45, 588-601. doi: 10.1002/hep. 21541 
Prieto, J., Melero, I., and Sangro, B. (2015). Immunological landscape and immunotherapy of hepatocellular carcinoma. Nat. Rev. Gastroenterol. Hepatol. 12, 681-700. doi: 10.1038/nrgastro.2015.173

Radziewicz, H., Ibegbu, C. C., Fernandez, M. L., Workowski, K. A., Obideen, K., Wehbim, M., et al. (2007). Liver-infiltrating lymphocytes in chronic human hepatitis $\mathrm{C}$ virus infection display an exhausted phenotype with high levels of PD-1 and low levels of CD127 expression. J. Virol. 81, 2545-2553. doi: 10.1128/JVI.02021-06

Riva, E., Scagnolari, C., Turriziani, O., and Antonelli, G. (2014). Hepatitis C virus and interferon type III (interferon-lambda3/interleukin-28B and interferonlambda4): genetic basis of susceptibility to infection and response to antiviral treatment. Clin. Microbiol. Infect. 20, 1237-1245. doi: 10.1111/1469-0691.12797

Ryerson, A. B., Eheman, C. R., Altekruse, S. F., Ward, J. W., Jemal, A., Sherman, R. L., et al. (2016). Annual report to the nation on the status of cancer, 19752012, featuring the increasing incidence of liver cancer. Cancer 122, 1312-1337. doi: $10.1002 /$ cncr.29936

Salmaninejad, A., Khoramshahi, V., Azani, A., Soltaninejad, E., Aslani, S., Zamani, M. R., et al. (2018). PD-1 and cancer: molecular mechanisms and polymorphisms. Immunogenetics 70, 73-86. doi: 10.1007/s00251-017-1015-5

Sansone, P., and Bromberg, J. (2012). Targeting the interleukin-6/Jak/stat pathway in human malignancies. J. Clin. Oncol. 20, 1005-1014. doi: 10.1200/JCO.2010. 31.8907

Sansonno, D., Carbone, A., De Re, V., and Dammacco, F. (2007). Hepatitis C virus infection, cryoglobulinaemia, and beyond. Rheumatology 46, 572-578. doi: 10.1093/rheumatology/kel425

Sasaki, H., Tatemaysu, T., Okuda, K., Moriyama, S., Yano, M., and Fujii, Y. (2014). PD-1 gene promoter polymorphisms correlate with a poor prognosis in nonsmall cell lung cancer. Mol. Clin. Oncol. 2, 1035-1042. doi: 10.3892/mco. 2014.358

Syedbasha, M., and Egli, A. (2017). Interferon lambda: modulating immunity in infectious diseases. Front. Immunol. 8:119. doi: 10.3389/fimmu.2017.00119

Tahoori, M. T., Pourfathollah, A. A., Akhlaghi, M., Daneshmandi, S., Nicknam, M. H., and Soleimanifar, N. (2011). Association of programmed cell death-1 (PDCD-1) gene polymorphisms with rheumatoid arthritis in Iranian patients. Clin. Exp. Rheumatol. 29, 763-767.

Taliani, G., Spaziante, M., Biliotti, E., Borro, M., Palazzo, D., Grieco, S., et al. (2013). IL28B gene polymorphisms and US liver fatty changes in patients who spontaneously cleared hepatitis C virus infection. PLoS One 8:e67301. doi: 10.1371/journal.pone.0067301

Tanaka, Y., Nishida, N., Sugiyama, M., Kurosaki, M., Matsuura, K., Sakamoto, N., et al. (2009). Genome-wide association of IL28B with response to pegylated interferon-alpha and ribavirin therapy for chronic hepatitis C. Nat. Genet. 41, 1105-1109. doi: 10.1038/ng.449

Tang, W., Chen, S., Chen, Y., Lin, J., Lin, J., Wang, Y., et al. (2017). Programmed death-1 polymorphisms is associated with risk of esophagogastric junction adenocarcinoma in the chinese han population: a case-control study involving 2,740 subjects. Oncotarget 8, 39198-39208. doi: 10.18632/oncotarget.17338
Tejeda, C., Broadaway, A. K., Ombrello, M. J., Brown, M. R., Ponder, L. A., Pichavant, M. R., et al. (2017). Case-control association study of autoimmunity associated variants in PDCD1 and juvenile idiopathic arthritis. Curr. Rheumatol. Rev. 13, 219-223. doi: 10.2174/1573397113666170104123113

Thomas, E., Gonzalez, V. D., Li, Q., Modi, A. A., Chen, W., Noureddin, M., et al. (2012). HCV infection induces a unique hepatic innate immune response associated with robust production of type III interferons. Gastroenterology 142, 978-988. doi: 10.1053/j.gastro.2011.12.055

Wack, A., Terczynska-Dyla, E., and Hartmann, R. (2015). Guarding the frontiers: the biology of type III interferons. Nat. Immunol. 16, 802-809. doi: 10.1038/ni. 3212

Wang, B. J., Bao, J. J., Wang, J. Z., Wang, Y., Jiang, M., Xing, M. Y., et al. (2011). Immunostaining of PD-1/PD-Ls in liver tissues of patients with hepatitis and hepatocellular carcinoma. World J. Gastroenterol. 17, 3322-3329. doi: 10.3748/ wjg.v17.i28.3322

Xiao, W., Zhang, Q., Deng, X. Z., Jiang, L. F., Zhu, D. Y., Pei, J. P., et al. (2015). Genetic variations of IL-28B and PD-1 are in association with the susceptibility and outcomes of HCV infection in southeast china. Infect. Genet. Evol. 32, 89-96. doi: 10.1016/j.meegid.2015.02.022

Yakut, M., Ozkan, H., Karakaya, F., and Erdal, H. (2018). Diagnostic and prognostic role of serum interleukin-6 in malignant transformation of liver cirrhosis. Eur. J. Hepatogastroenterol. 8, 23-30. doi: 10.5005/jp-journals-10018-1253

Zhang, G., Li, N., Li, Z., Zhu, Q., Li, F., Yang, C., et al. (2015). microRNA-4717 differentially interacts with its polymorphic target in the PD1 3' untranslated region: a mechanism for regulating PD-1 expression and function in HBVassociated liver diseases. Oncotarget 6, 18933-18944. doi: 10.18632/oncotarget. 3662

Zhang, G., Liu, Z., Duan, S., Han, Q., Li, Z., Lv, Y., et al. (2010). Association of polymorphisms of programmed cell death-1 gene with chronic hepatitis $B$ virus infection. Hum. Immunol. 71, 1209-1213. doi: 10.1016/j.humimm.2010. 08.014

Zhou, J., Wang, Y., Chang, Q., Ma, P., Hu, Y., and Cao, X. (2018). Type III interferons in viral infection and antiviral immunity. Cell Physiol. Biochem. 51, 173-185. doi: 10.1159/000495172

Conflict of Interest Statement: The authors declare that the research was conducted in the absence of any commercial or financial relationships that could be construed as a potential conflict of interest.

Copyright (C) 2019 De Re, Tornesello, De Zorzi, Caggiari, Pezzuto, Leone, Racanelli, Lauletta, Gragnani, Buonadonna, Vaccher, Zignego, Steffan and Buonaguro. This is an open-access article distributed under the terms of the Creative Commons Attribution License (CC BY). The use, distribution or reproduction in other forums is permitted, provided the original author(s) and the copyright owner(s) are credited and that the original publication in this journal is cited, in accordance with accepted academic practice. No use, distribution or reproduction is permitted which does not comply with these terms. 